\title{
The role of EEG gamma frequency in shooting performance
}

Article in International Journal of Psychophysiology · September 2010

DOI: 10.1016/j.ijpsycho.2010.06.183

CITATIONS

0

2 authors:

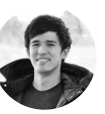

\section{Ming-Yang Cheng}

Bielefeld University

23 PUBLICATIONS 5 CITATIONS

SEE PROFILE
READS

13

Some of the authors of this publication are also working on these related projects:

Project

Exercise and executive function in children with ADHD View project

The Effect of SMR Neurofeedback Training on Mental Representation and Golf Putting Performance

Project

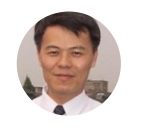

Tsung-Min Hung

National Taiwan Normal University

140 PUBLICATIONS 508 CITATIONS

SEE PROFILE 
explained by the use of different cue types than previously reported findings. Additionally, the spatial cueing task may not elicit a P3 component which permits nicotine modulation.

doi:10.1016/j.ijpsycho.2010.06.180

\section{The evaluation of learning via event-related tactile potentials}

Adile Oniz, Cagdas Guducu, Onur Bayazit, Murat Ozgoren Department of Biophysics, Faculty of Medicine, Dokuz Eylul University, Izmir, 35340, Turkey

The aim of the study was to assess possible learning effects on the sensory and cognitive components using a tactile paradigm in two consecutive sessions. Twenty-three subjects were recruited into the study ( $21.44 \pm 2.73$ years; 8 females) for the electrophysiological analysis of 64 channel EEG data. Simple (mechanical) tactile stimuli were delivered using a 4-D pneumatic stimulator, delivering a soft touch stimulus on to the index (target) and middle fingers (nontarget) of the subjects in two consecutive sessions. The responses to the tactile stimuli were observed widely across the electrode sites, yet the $\mathrm{Cz}$ (central) electrode showed the highest amplitudes. These responses were grouped into three time windows and their respective waveforms were measured. The analysis revealed that very early waveforms (50-120 ms) remained the same in two sessions, whereas, the early responses $(150-250 \mathrm{~ms})$ decreased significantly from the first session to the second (for each comparison, $p<0.05$ ). The late time components (300-450 ms) maintained their levels throughout the experiment. The target and standard late responses were also found to be significantly different in two consecutive sessions (for each comparison, $p<0.001$ ). The very early responses remaining about the same in two sessions may be related to the nature of sensory responses. The decreasing of early responses can be explained by the attendance level of the subjects, thus pointing to their levels of learning the template of the experimental setup. Consequently, consistency of the late response has been related to the task performance of the subjects, where they are required to maintain their attention throughout the experiment. The results point to an applicable model to study neurocognitive, as well as other clinical cases (ie.vasculomotor).

doi:10.1016/j.ijpsycho.2010.06.181

\section{Differences of alpha power in the occipital cortex among different skill levels}

Yu-Jung Tsai, Tai-Ting Chen, Tsung-Min Hung

National Taiwan Normal University, Department of Physical Education, Taipei, Taiwan

The purpose of this study was to examine alpha power differences in the occipital cortex among subjects from three different skill levels in darts throw. Sixty-nine participants (18-50 years), 17 experts, 22 intermediates and 30 novices, took part in 60 darts throwing tests with brain waves recorded $(4 \times 0.5 \mathrm{~s}$ epochs $)$ in their occipital region during aiming. Two factors from mixed design variance analysis were used to compare the differences in occipital region among three different skill level groups. The results showed that there was no interaction between three groups and four epochs in occipital alpha, which means that there was no difference between the three groups during aiming. However, there was a significant main effect for group and time. The alpha power in experts was higher than in the others. Moreover, the alpha power in the three groups was higher in the first epoch than the second and third epoch $(p<0.05)$. Nevertheless, in the power there was no difference between the first and the fourth epochs, which reflected that the alpha power in these three groups was higher in the first and the fourth epoch. In conclusion, the alpha power increased in the occipital region among those from the expert group before dart throw indicating less visual attention, which supports past studies about expert's model of automaticity in that the expert's task procedure as a parallel model doesn't need additional attention source but faster processing.

doi:10.1016/j.ijpsycho.2010.06.182

\section{The role of EEG gamma frequency in shooting performance}

Ming Yang Cheng, Tsung Min Hung

National Taiwan Normal University, Department of Physical Education, Taipei, Taiwan

$\gamma(36-44 \mathrm{~Hz})$ rhythms are an index of brain activation. According to concurrent EEG and PET measurements, $\gamma$ rhythms have the highest number of positive correlations between current density and glucose metabolism. Furthermore, $\gamma$ rhythms are associated with attention, arousal, object recognition and perceptual binding. However, there have been few studies examining the relationship between $\gamma$ rhythms and precision sport performance, leading to an absence of understanding about $\gamma$ rhythm activation during tasks requiring high levels of attention and stability (e.g. shooting). Therefore, this study hypothesized that $\gamma$ rhythms play a critical role in shooting performance and are an indicator of attention during the aiming time (AT) before shooting. For the purposes of this study it was assumed that the attention process before skill execution is composed of several continuous mental phases. Twenty-three elite shooters were recruited, with EEG data of the best and worst five shots for each participant divided into three parts based on AT. In order to examine the different attentional states between best and worst performances among the respective AT, $\gamma$ rhythm served as the dependent variable representing the activation of the brain. $\Gamma$ rhythm was examined at all lobes. Several 2 (performance) $\times 3$ (epoch) $\times 18$ (electrode) ANOVA with repeated measures were utilized to examine the relationship between shooting performance and EEG activity of $\gamma$. As predicted, $\gamma$ rhythms of best performance AT were higher than the worst in all brain regions. Additionally, $\gamma$ rhythms gradually decreased when approaching trigger pull. No such interaction between performance and time factors was detected. These results suggest that greater attention and perceptual binding involved during AT facilitated the shooting performance. Less $\gamma$ rhythms in the cerebral cortical activity preceding trigger pull were associated with the requirement for executing the shooting task and likely a mechanism of neural efficiency. $\gamma$ reduction in each separate AT approaching trigger pull indicates that the complete preparatory period of shooting tasks has a similar process in sequence.

doi:10.1016/j.ijpsycho.2010.06.183

\section{Examining attention-related EEG differences between best and the worst shooting performances in consideration of separate aiming times}

Shih-Chun Kao, Tsung-Min Hung

National Taiwan Normal University, Department of Physical Education, Taipei, Taiwan

Purpose: Greater T3 $\alpha, \mathrm{Oz} \alpha, \mathrm{Fz} \theta$ and lower $\mathrm{Cz} \alpha 2$ power prior to skill execution have been found to be related to an optimal 